\title{
Dynamics of inertial vortices in multi-component Bose-Einstein condensates
}

\author{
Katsuhiro Nakamura ${ }^{(1,2)}$, Doniyor Babajanov ${ }^{(1)}$, Davron Matrasulov ${ }^{(3)}$, Michikazu Kobayashi ${ }^{(4)}$ \\ ${ }^{(1)}$ Faculty of Physics, National University of Uzbekistan, Vuzgorodok, Tashkent 100174,Uzbekistan \\ ${ }^{(2)}$ Department of Applied Physics, Osaka City University, Osaka 558-8585, Japan \\ ${ }^{(3)}$ Turin Polytechnic University in Tashkent, 17 Niyazov Str., Tashkent 100093, Uzbekistan \\ (4) Department of Basic Science, University of Tokyo, Tokyo 153-8902, Japan
}

(Dated: November 9, 2018)

\begin{abstract}
With use of the nonlinear Schrödinger (or Gross-Pitaevskii) equation with strong repulsive cubic nonlinearity, dynamics of multi-component Bose-Einstein condensates (BECs) with a harmonic trap in 2 dimensions is investigated beyond the Thomas-Fermi regime. In the case when each component has a single vortex, we obtain an effective nonlinear dynamics for vortex cores (particles). The particles here acquire the inertia, in marked contrast to the standard theory of point vortices widely known in the usual hydrodynamics. The effective dynamics is equivalent to that of charged particles under a strong spring force and in the presence of Lorentz force with the uniform magnetic field. The inter-particle (vortex-vortex) interaction is singularly-repulsive and short-ranged with its magnitude decreasing with increasing distance of the center of mass from the trapping center. "Chaos in the three-body problem" in the three vortices system can be seen, which is not expected in the corresponding point vortices without inertia in 2 dimensions.
\end{abstract}

PACS numbers: 03.75.-b, 05.45.-a, 05.60.Gg.

\section{INTRODUCTION}

Recently, there has been much interest in theoretical and experimental studies on trapped atomic BoseEinstein condensates (BECs) [1] 3 . The superfluid property of atomic BECs arises from a dual aspect of waves and particles, i.e., matter waves, and is theoretically described by the macroscopic wave function. Because of the nonlinearity of the system caused by interaction between particles, the macroscopic wave function can take a form of various solitons such as bright, dark, grey, and vortex solitons, and these solitons are experimentally observed in BECs.

As for bright solitons, Martin et al. [4] theoretically predicted that the particle-like behavior of three bright solitons in a one-dimensional ${ }^{87} \mathrm{Rb} \mathrm{BEC}$ was non-integrable and showed its change from regular motions to chaos. Pérez-García et al. [5] applied a variational method to dynamics of bright solitons in a two-dimensional (2-d) BEC and showed that the center of mass of each soliton obeys Newtonian dynamics and Ehrenfest's theorem is valid if the phase of BEC wave function will be suitably chosen.

As more interesting systems, we can consider solitons in multi-component BECs which consist of different kinds of atoms or same kinds of atoms having different spin and have been experimentally realized. In multicomponent BECs, there are not only intra-component particle interaction but also inter-component particle in- teraction which is another origin of nonlinearity, so we expect novel soliton dynamics which is not seen in singlecomponent BECs. Yamasaki et al. [6] ] developed a variational method to describe bright soliton dynamics in 2-d multi-components BECs, and proposed a model of conservative chaos. In 2-d and 3-d systems, however, bright soliton are unstable unless intra-component interaction oscillates between attraction and repulsion or coexisting intra-component quintic (three-body) interaction is strong enough.

On the other hand, topological vortices known as quantized vortices, i.e., topological defects of the macroscopic wave function can be stable in two dimensions. Vortices in single-component [7 9] and multi-component [1012. BECs have been realized experimentally, giving a good candidate to study the dynamics of vortices in 2-d and 3-d BECs. Most of the theoretical studies, however, are limited to the Thomas-Fermi regime (TFR) in a single-component BEC [9, 13 15]. While dynamics of the macroscopic wave function of BEC is described by Gross-Pitaevskii equation (GPE) in Eq.(2) below, TFR suppresses the kinetic energy part in GPE in the lowest approximation and therefore the lowest-order wave function cannot have a healing length which is a hallmark of the vortex core. It is highly desirable to construct the effective theory of vortices beyond the TFR.

In this paper, we consider the vortices in 2-d multicomponent BECs beyond the Thomas-Fermi regime. To 
consider the effective dynamics of point-like vortices, we extract some degrees of freedom by using the variational approach, and derive an effective dynamics with finite degrees of freedom. For the case of a single-component BEC without a trap, it is well known that the effective hamiltonian for many vortex systems has a standard form [16 18

$$
H \sim \sum_{j>i} n_{i} n_{j} \ln r_{i j}
$$

in the limit of infinitesimal vortex cores. Here $n_{i}$ is the winding number of the $i$-th vortex, and $r_{i j}=$ $\sqrt{\left(x_{i}-x_{j}\right)^{2}+\left(y_{i}-y_{j}\right)^{2}}$ is the distance between cores of $i$-th and $j$-th vortices. Equation (11) shows that there is no momentum degree of freedom, and coordinates $x_{i}$ and $y_{i}$ are formally conjugate each other. On the other hand, in the case of multi-component BECs in a trap with each component having a single vortex, we shall see vortices acquire momentum degrees of freedom or inertia. A very preliminary idea of the present work was reported in the conference proceedings [19].

This paper is organized as follows. In Sec. III starting from the multi-component GPE, we apply the variational method with use of vortex solitons in the Padé approximation, and derive the effective Hamiltonian for vortices. Confining to the case of two and three vortices, we numerically investigate the detailed dynamics of vortices in Sec. III. There we see a chaotic behavior of the system consisting of three vortices. Section IV is devoted to conclusions and discussions.

\section{EFFECTIVE NONLINEAR DYNAMICS GENERATED BY THE MULTI-COMPONENT GPE}

In this Section, we consider the trapped multicomponent GPE with vortices and extract some degrees of freedom of vortex soliton by using a variational technique.

BEC at zero temperature is described by the GPE. We shall consider a 2-d system of trapped $n$-component macroscopic wave function $\Phi_{1}(t, x, y), \Phi_{2}(t, x, y), \cdots, \Phi_{n}(t, x, y) \quad$ satisfying the equations

$$
\begin{aligned}
i \frac{\partial}{\partial t} \Phi_{i}(t, x, y) & =\left[-\nabla^{2}+V(x, y)+g_{i i}\left|\Phi_{i}(t, x, y)\right|^{2}\right. \\
& \left.+\sum_{j \neq i} g_{i j}\left|\Phi_{j}(t, x, y)\right|^{2}\right] \Phi_{i}(t, x, y),
\end{aligned}
$$

for $i, j=1, \ldots, n$. Here the normalization condition for each component of wave functions is defined by $\int\left|\Phi_{i}(t, x, y)\right|^{2} d x d y=1$ after a proper rescaling of $\Phi_{i}$ by the particle number $N$ common to all components. The effect of trapping is expressed by $V(x, y)=\left(x^{2}+y^{2}\right)$. Equation (2) is expressed with use of scaled variables: using the confining length $l=\sqrt{\frac{\hbar}{m \omega}}$ and oscillation pe$\operatorname{riod} \tau=\omega^{-1}$, space coordinates are scaled by $l$, time by $2 \tau$, wave function by $\frac{1}{l}$, and nonlinearity by $\frac{\hbar^{2}}{2 m}$. The nonlinearity coefficients, $g_{i j} \equiv 8 \pi N a_{i j} / l$ with $a_{i j}$ the scattering length for binary collisions, are assumed to be positive and much larger than unity. $g_{i i}$ and $g_{i j}$ with $i \neq j$ stand for intra-component and inter-component interactions, respectively. We shall choose $g_{i i}=g_{1}(\gg 1)$ for all $i$ and $g_{i j}=g_{2}(\gg 1)$ for $i \neq j$.

In the absence of the inter-component interaction, each component has stationary states of a vortex. So, we consider the case in which each component has one vortex and vortices interact with each other through the intercomponent interaction. Our goal is to derive from (2) the evolution equation for the collective coordinates of trial vortex functions (TVFs). The collective coordinates for a vortex are phase variables besides the coordinates of a vortex core. We shall use TVF beyond the ThomasFermi regime, by incorporating the effect of a kinetic energy in GPE in Eq.(2): As for the amplitude of TVF, we choose a vortex function based on the Padé approximation [20, 21] which is regularized due to a trap. As for its phase, we Taylor-expand the phase with respect to space coordinates around the vortex core. Then TVF with winding number $n_{i}= \pm 1$ is given by

$$
\begin{aligned}
& \Phi_{i}(t, x, y) \equiv f_{i}(t, x, y) \exp \left[i \phi_{i}(t, x, y)\right] \\
& =N \exp \left[-\frac{x^{2}+y^{2}}{2 \Delta}\right] \sqrt{\frac{\left(x-x_{i}\right)^{2}+\left(y-y_{i}\right)^{2}}{2 \xi^{2}+\left(x-x_{i}\right)^{2}+\left(y-y_{i}\right)^{2}}} \\
& \times \exp \left[i\left[n_{i} \tan ^{-1}\left(\frac{y-y_{i}}{x-x_{i}}\right)+\alpha_{i}\left(x-x_{i}\right)+\beta_{i}\left(y-y_{i}\right)\right]\right]
\end{aligned}
$$

with the normalization factor ${ }^{N}=$
$\sqrt{\pi \Delta-2 \pi e^{\frac{2 \xi^{2}}{\Delta}} \xi^{2} \Gamma\left(0, \frac{2 \xi^{2}}{\Delta}\right)}$
is the incomplete gamma function of the second kind, is the incomplete gamma function of the second kind,
whose expansion with respect to $z$ is given in Eq.A10 in Appendix $\mathrm{A}$

The collective coordinates are locations of the core $\left(x_{i}, y_{i}\right)$ and the first-order coefficients $\left(\alpha_{i}, \beta_{i}\right)$ of Taylor- 
expansion of the phase $\phi_{i}(t, x, y)$ with respect to $(x-$ $\left.x_{i}, y-y_{i}\right) . \Delta$ in the Gaussian amplitude factor reflects a trap in Eq.(3). $\quad \xi$ is the healing length related to vortex core size. The condition to minimize the energy $E=\int d x d y\left(\left|\nabla \Phi_{i}\right|^{2}+V(x, y)\left|\Phi_{i}\right|^{2}+\frac{g_{1}}{2}\left|\Phi_{i}\right|^{4}\right)$ for the individual static component in Eq.(3) centered at the origin, leads to $\Delta \cong \sqrt{\frac{3}{2}-(\gamma+1) n_{i}^{2}+\frac{g_{1}}{4 \pi}}$ and $\xi \cong \frac{\left|n_{i}\right| \pi^{1 / 4}}{\sqrt{2+\gamma}} g_{1}^{-1 / 4}$, respectively, where $\gamma(=0.57721)$ is Euler constant. We shall use these values for $\Delta$ and $\xi$ in this paper.

The form in Eq.(3), which is a product of the vortex solution in the absence of a harmonic trap and Gaussian factor due to the trap, gives a suitable TVF for a vortex under the strong nonlinearity. A different form with use of eigenstates (with non-zero angular momenta) under the 2-d harmonic trap [22 24] has no small healing length, results in the inter-vortices force growing with inter-vortices distance, etc, and cannot be suitable as TVF under the strong nonlinearity.

First of all we note: GPE in Eq.(2) can be derived from the variational principle that minimizes the action obtained from Lagrangian density $\mathcal{L}$ for field variables,

$$
\begin{aligned}
-\mathcal{L}= & \sum_{i}\left[\frac{i}{2}\left(\Phi_{i} \dot{\Phi}_{i}^{*}-\Phi_{i}^{*} \dot{\Phi}_{i}\right)+\left|\nabla \Phi_{i}\right|^{2}\right. \\
& \left.+\left(x^{2}+y^{2}\right)\left|\Phi_{i}\right|^{2}+\frac{g_{1}}{2}\left|\Phi_{i}\right|^{4}\right]+\sum_{j>i} g_{2}\left|\Phi_{i}\right|^{2}\left|\Phi_{j}\right|^{2}
\end{aligned}
$$

In fact, Eq.(2) is obtained from Lagrange equation:

$$
\frac{\partial}{\partial t} \frac{\partial \mathcal{L}}{\partial \dot{\Phi}_{i}^{*}}-\frac{\partial \mathcal{L}}{\partial \Phi_{i}^{*}}+\nabla \frac{\partial \mathcal{L}}{\partial \nabla \Phi_{i}^{*}}=0
$$

We now insert TVF in Eq. (3) into Eq. (4). Noting $\left(x_{i}, y_{i}\right)$ and $\left(\alpha_{i}, \beta_{i}\right)$ as time-dependent variables, Eq. (4) becomes

$$
\begin{aligned}
-\mathcal{L} & =\sum_{i}\left[\left(\dot{x}_{i} \frac{\partial \phi_{i}}{\partial x_{i}}+\dot{y}_{i} \frac{\partial \phi_{i}}{\partial y_{i}}+\dot{\alpha}_{i} \frac{\partial \phi_{i}}{\partial \alpha_{i}}+\dot{\beta}_{i} \frac{\partial \phi_{i}}{\partial \beta_{i}}\right) f_{i}^{2}\right. \\
& +\left(\frac{\partial f_{i}}{\partial x}\right)^{2}+\left(\frac{\partial f_{i}}{\partial y}\right)^{2}+\left(\left(\frac{\partial \phi_{i}}{\partial x}\right)^{2}+\left(\frac{\partial \phi_{i}}{\partial y}\right)^{2}\right) f_{i}^{2} \\
& \left.+\frac{g_{1}}{2} f_{i}^{4}+\left(x^{2}+y^{2}\right) f_{i}^{2}\right]+g_{2} \sum_{j>i} f_{i}^{2} f_{j}^{2}
\end{aligned}
$$

By integrating $\mathcal{L}$ over space coordinates $(x, y)$, we obtain the effective Lagrangian $L$ for the collective coordinates:

$$
L=\iint d x d y \mathcal{L}
$$

In the limit of $\xi^{2} / \Delta \ll 1, L$ is expressed by

$$
\begin{aligned}
- & L=\left(\frac{N}{N_{0}}\right)^{2} \sum_{i}\left[-\frac{n_{i}}{\Delta}\left(\dot{x}_{i} y_{i}-\dot{y}_{i} x_{i}\right) e^{-\frac{l_{i}^{2}}{\Delta}}\left(d_{1}+\frac{d_{2} l_{i}^{2}}{\Delta}\right)\right. \\
& +\left(\alpha_{i}^{2}+\beta_{i}^{2}-\left(\alpha_{i} \dot{x}_{i}+\beta_{i} \dot{y}_{i}\right)\right) d_{1} \\
& -\left(\dot{\alpha}_{i} x_{i}+\dot{\beta}_{i} y_{i}\right)\left(1-\frac{2 \xi^{2}}{\Delta}\left(1-\frac{l_{i}^{2}}{2 \Delta}\right)\right) \\
& +\frac{1}{\Delta} e^{-\frac{l_{i}^{2}}{\Delta}}\left(2 n_{i}^{2} d_{3}+d_{4}+2 n_{i}^{2} \frac{l_{i}^{2} d_{5}}{\Delta}+d_{6} \frac{l_{i}^{2}}{\Delta}\right) \\
& +2 \frac{n_{i}}{\Delta}\left(y_{i} \alpha_{i}-x_{i} \beta_{i}\right) e^{-\frac{l_{i}^{2}}{\Delta}}\left(d_{1}+\frac{l_{i}^{2}}{\Delta} d_{2}\right) \\
& +e^{-\frac{l_{i}^{2}}{\Delta}}\left(d_{8}+l_{i}^{2} d_{9}\right) \\
+ & \left.\left(\frac{N}{N_{0}}\right)^{2} \frac{g_{1}}{\pi \Delta}\left(\frac{1}{4}+d_{7} e^{-\frac{2 l_{i}^{2}}{\Delta}}\right)\right] \\
+ & \sum_{j>i} U\left(r_{i j}, l_{G}^{i j}\right) .
\end{aligned}
$$

Here, $l_{i}=\sqrt{x_{i}^{2}+y_{i}^{2}}$ and $N_{0}=\frac{1}{\sqrt{\pi \Delta}}$. Expressions $($ : Taylor-expansions with respect to $\xi^{2} / \Delta$ ) for coefficients $d_{1} \sim d_{9}$ are listed in Table 1 and the derivation of typical coefficients is described in Appendix A. The expression for the inter-vortices interaction $U\left(r_{i j}, l_{G}^{i j}\right)$, which is a function of inter-vortices distance $r_{i j}=\sqrt{x_{i j}^{2}+y_{i j}^{2}}$ and distance of the center of masses from the origin $l_{G}^{i j}=$ $\sqrt{\left(x_{G}^{i j}\right)^{2}+\left(y_{G}^{i j}\right)^{2}}$, will be given in Appendix B.

Lagrange equations of motion for the phase variables $\alpha_{i}$ and $\beta_{i}$,

$$
\begin{aligned}
& \frac{d}{d t}\left(\frac{\partial L}{\partial \dot{\alpha}_{i}}\right)-\frac{\partial L}{\partial \alpha_{i}}=0 \\
& \frac{d}{d t}\left(\frac{\partial L}{\partial \dot{\beta}_{i}}\right)-\frac{\partial L}{\partial \beta_{i}}=0
\end{aligned}
$$

lead to

$$
\begin{aligned}
& \alpha_{i} \simeq B_{1} \dot{x}_{i}-n_{i} B_{2} y_{i} \\
& \beta_{i} \simeq B_{1} \dot{y}_{i}+n_{i} B_{2} x_{i}
\end{aligned}
$$

Equation (10) shows that $\left(\alpha_{i}, \beta_{i}\right)$ correspond to generalized momentum conjugate to $\left(x_{i}, y_{i}\right)$ under the vector potential. Then the equation of motion for $\left(x_{i}, y_{i}\right)$

$$
\begin{aligned}
& \frac{d}{d t}\left(\frac{\partial L}{\partial \dot{x}_{i}}\right)-\frac{\partial L}{\partial x_{i}}=0 \\
& \frac{d}{d t}\left(\frac{\partial L}{\partial \dot{y}_{i}}\right)-\frac{\partial L}{\partial y_{i}}=0
\end{aligned}
$$


combined with Eq.(10), gives

$$
\begin{aligned}
& \ddot{x}_{i} \simeq n_{i} B_{3} \dot{y}_{i}-B_{4} x_{i}-B_{5} \sum_{j \neq i} \frac{\partial U\left(x_{i j}, y_{i j}\right)}{\partial x_{i}} \\
& \ddot{y}_{i} \simeq-n_{i} B_{3} \dot{x_{i}}-B_{4} y_{i}-B_{5} \sum_{j \neq i} \frac{\partial U\left(x_{i j}, y_{i j}\right)}{\partial y_{i}} .
\end{aligned}
$$

Table 1. Expressions for coefficients in Eq.(8) (: Taylor-expansions with respect to $\xi^{2} / \Delta$ ) .

\begin{tabular}{|c|c|}
\hline Coefficients & Expressions \\
\hline$d_{1}$ & $1+\frac{2 \gamma \xi^{2}}{\Delta}+\frac{2 \xi^{2}}{\Delta} \ln \frac{2 \xi^{2}}{\Delta}$ \\
\hline$d_{2}$ & $\frac{1}{2}-\frac{\xi^{2}}{\Delta}$ \\
\hline$d_{3}$ & $-\frac{\gamma}{2}-\frac{1}{2} \ln \frac{2 \xi^{2}}{\Delta}+\frac{\xi^{2}}{\Delta}-\frac{\gamma \xi^{2}}{\Delta}-\frac{\xi^{2}}{\Delta} \ln \frac{2 \xi^{2}}{\Delta}$ \\
\hline$d_{4}$ & $\frac{3}{2}+\frac{\xi^{2}}{\Delta}+\frac{4 \gamma \xi^{2}}{\Delta}+\frac{4 \xi^{2}}{\Delta} \ln \frac{2 \xi^{2}}{\Delta}$ \\
\hline$d_{5}$ & $\frac{1}{2}+\frac{\gamma \xi^{2}}{\Delta}+\frac{\xi^{2}}{\Delta} \ln \frac{2 \xi^{2}}{\Delta}$ \\
\hline$d_{6}$ & $1+\frac{5 \xi^{2}}{\Delta}+\frac{6 \gamma \xi^{2}}{\Delta}+\frac{6 \xi^{2}}{\Delta} \ln \frac{2 \xi^{2}}{\Delta}$ \\
\hline$d_{7}$ & $\frac{\xi^{2}}{\Delta}+\frac{2 \gamma \xi^{2}}{\Delta}+\frac{2 \xi^{2}}{\Delta} \ln \frac{4 \xi^{2}}{\Delta}$ \\
\hline$d_{8}$ & $\Delta-2 \xi^{2}$ \\
\hline$d_{9}$ & $1+\frac{2 \xi^{2}}{\Delta}+\frac{2 \gamma \xi^{2}}{\Delta}+\frac{2 \xi^{2}}{\Delta} \ln \frac{2 \xi^{2}}{\Delta}$ \\
\hline
\end{tabular}

Coefficients $B_{1} \sim B_{5}$ are given by

$$
\begin{aligned}
& B_{1}=\frac{d_{1}-1+\frac{2 \xi^{2}}{\Delta}}{2 d_{1}} \sim \frac{(\gamma+1) \xi^{2}}{\Delta} \\
& B_{2}=\frac{1}{\Delta} \\
& B_{3}=-\frac{4 d_{1}^{2}}{\Delta\left(d_{1}-1+\frac{2 \xi^{2}}{\Delta}\right)^{2}} \sim-\frac{\Delta}{(\gamma+1)^{2} \xi^{4}} \\
& B_{4}=\frac{4 d_{1} d_{9}}{\left(d_{1}-1+\frac{2 \xi^{2}}{\Delta}\right)^{2}} \sim \frac{\Delta^{2}}{(\gamma+1)^{2} \xi^{4}} \\
& B_{5}=\left(\frac{N}{N_{0}}\right)^{2} \frac{2 d_{1}}{\left(d_{1}-1+\frac{2 \xi^{2}}{\Delta}\right)^{2}} \sim \frac{\Delta^{2}}{2(\gamma+1)^{2} \xi^{4}} .
\end{aligned}
$$

Equations (10) and (13) are valid aside from a multiplicative global factor $\left(1+O\left(\frac{l_{i}^{2}}{\Delta}\right)\right)$. The smallness of $\frac{l_{i}^{2}}{\Delta}$ will be justified a posteriori. Equation (13) shows that dynamics of coordinates $\left(x_{i}, y_{i}\right)$ is very similar to charged particles with charges $n_{i}= \pm 1$ under a strong spring force with a force constant $B_{4}=O\left(g_{1}^{2}\right)$ and in the presence of Lorentz force with the magnetic field $\mathbf{B}=\left(0,0, B_{3}\right)$. One should note that the spring force here has nothing to do with that of the original harmonic potential $V(x, y)$ in Eq.(2). The Hamiltonian corresponding to Eqs. (13) can be given by

$$
H=\sum_{i}\left[\frac{1}{2 m}\left[\mathbf{p}_{i}-n_{i} \mathbf{A}_{i}\right]^{2}+W_{i}+\sum_{j>i} \tilde{U}\left(r_{i j}, l_{G}^{i j}\right)\right],
$$

with use of the momentum $\mathbf{p}_{i} \equiv m \dot{\mathbf{r}}+n_{i} \mathbf{A}_{i}$, unit mass $m=1$, the vector potential $\mathbf{A}_{i}=\frac{B_{3}}{2}\left(-y_{i}, x_{i}\right)$, and the scalar potential $W_{i}=\frac{1}{2} B_{4}\left(x_{i}^{2}+y_{i}^{2}\right)$.

All values $B_{1} \sim B_{5}$ depend on the strength of interaction $g_{1}$ which is tunable by Feshbach resonance. In the effective particle dynamics described by Eqs.(13) and (15), the spring constant $B_{4}$ is large enough to guarantee particles with unit mass to be confined in the neighborhood of the origin, i.e., the trapping center. This finding justifies $\frac{l_{i}^{2}}{\Delta} \ll 1$ and will also be utilized to obtain the inter-vortices interaction in Appendix B.

The scaled inter-vortices interaction $\tilde{U}\left(r_{i j}, l_{G}^{i j}\right)$ is given with use of Eq.(B10) as

$$
\begin{aligned}
\tilde{U}\left(r_{i j}, l_{G}^{i j}\right) & =B_{5} U_{4}\left(r_{i j}, l_{G}^{i j}\right) \\
& \cong \frac{G}{r_{i j}^{2}} \exp \left(-\frac{r_{i j}^{2}+4\left(l_{G}^{i j}\right)^{2}}{2 \Delta}\right)
\end{aligned}
$$

with the coupling constant

$$
G=\frac{4 g_{2}}{(\gamma+1)^{2} \pi^{1 / 2}} .
$$

Therefore the inter-vortices interaction is singularlyrepulsive and short-ranged with respect to $r_{i j}$ with its magnitude decreasing with increasing $l_{G}^{i j}$.

Compared to Eq. (1), it is clear that the system has momentum degrees of freedom, and vortices have a behavior of particle with inertia rather than that of vortex 
point without inertia widely used in the conventional theory of hydrodynamics [25 29]. This is one of the main assertions of the present paper. The inertia of vortex appears already in the single-component BEC with a trap.

In closing this Section we should comment: we also attempted to apply the collective coordinate method using a Laguerre-type trial function which is a good candidate for TVF in the case of a weak nonlinearity 22 24]. However, such TVF in the case of a strong nonlinearity proved to result in: 1) a time-dependent mass for each vortex and 2) the inter-vortex interaction growing with increasing the inter-vortex distance. Hence this TVF was not suitable to describe a dynamics of vortices in BECs with a strong nonlinearity. On the other hand, the effective vortex dynamics in the Thomas-Fermi regime [9, 13 15], which suppresses the kinetic energy in constructing TVF, leads to neither non-zero inertia nor Lorentz force.

\section{DYNAMICS OF TWO AND THREE VORTICES WITH INERTIA}

We shall now focus on the system of two vortices with equal winding numbers, and see how the trajectory generated by effective particle dynamics in Eq.(13) and (15) well mimics the orbit of the singular points of wave vortices calculated by using GPE in Eq.(2). We shall then move to the system of three vortices with equal winding numbers, and find that chaos appears even in the three vortex system. This feature is different from that of point vortices system in a single component BEC in which chaos can appear in the case of more than three vortices.

\section{A. Dynamics of two vortices}

For two vortices with the same winding numbers $n_{1}=$ $n_{2}=1$, Hamiltonian (15) can be rewritten as

$$
\begin{aligned}
H & =\frac{1}{2}\left(p_{x, T}^{2}+p_{y, T}^{2}+p_{x, R}^{2}+p_{y, R}^{2}\right) \\
& -\frac{B_{3}}{2}\left(p_{x, T} y_{T}-p_{y, T} x_{T}+p_{x, R} y_{R}-p_{y, R} x_{R}\right) \\
& +\left(\frac{B_{3}^{2}}{8}+\frac{B_{4}}{2}\right)\left(x_{T}^{2}+y_{T}^{2}+x_{R}^{2}+y_{R}^{2}\right) \\
& +\frac{G}{2\left(x_{R}^{2}+y_{R}^{2}\right)} \exp \left(-\frac{x_{R}^{2}+y_{R}^{2}+x_{T}^{2}+y_{T}^{2}}{\Delta}\right)
\end{aligned}
$$

where $\left(x_{T}, y_{T}\right)$ and $\left(x_{R}, y_{R}\right)$ play the role of the center-of-mass and relative coordinates, respectively and

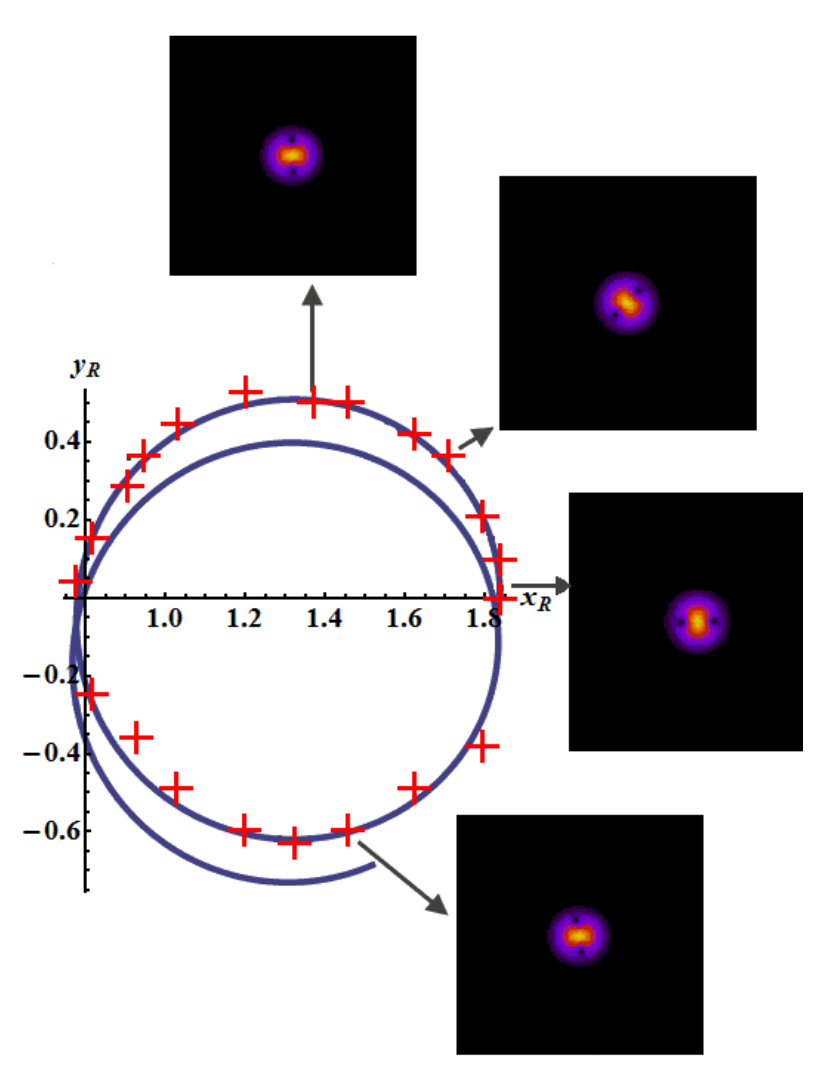

FIG. 1: Two vortices dynamics with identical winding numbers $\left(n_{1}=n_{2}=1\right) . \quad g_{1}=g_{2}=100 . \quad$ Initial values: $x_{1}(0)=y_{1}(0)=-x_{2}(0)=-y_{2}(0)=\frac{1}{\sqrt{2}} ; \dot{x_{1}}(0)=\dot{x_{2}}(0)=0$; $\dot{y}_{1}(0)=-\dot{y_{2}}(0)=1.1$. Solid line is a trajectory for relative coordinates of a pair of particles calculated by iterating the canonical equations for the Hamiltonian (18); crosses are corresponding results for a pair of singular points of interacting vortices constructed from the numerical iteration of GPE in Eq.(2); four sub-panels are wave function patterns for interacting vortices.

$\left(p_{x, T}, p_{y, T}\right)$ and $\left(p_{x, R}, p_{y, R}\right)$ are their canonical-conjugate variables. To be explicit,

$$
\begin{aligned}
\left(x_{T}, y_{T}\right) & =\frac{1}{\sqrt{2}}\left(x_{1}+x_{2}, y_{1}+y_{2}\right) \\
\left(x_{R}, y_{R}\right) & =\frac{1}{\sqrt{2}}\left(x_{1}-x_{2}, y_{1}-y_{2}\right) \\
\left(p_{x, T}, p_{y, T}\right) & =\frac{1}{\sqrt{2}}\left(p_{x, 1}+p_{x, 2}, p_{y, 1}+p_{y, 2}\right) \\
\left(p_{x, R}, p_{y, R}\right) & =\frac{1}{\sqrt{2}}\left(p_{x, 1}-p_{x, 2}, p_{y, 1}-p_{y, 2}\right) .
\end{aligned}
$$

Hamiltonian (15) cannot be reduced to two independent two degree-of-freedom subsystems (: center-ofmass system and relative-coordinates system) because the inter-vortex interaction depends not only on $r_{i j}$ but 
also on $l_{G}^{i j}$. However, we see mostly KAM tori in this system. As shown in Fig. 1, we find that the trajectory of $\left(x_{R}, y_{R}\right)$ generated by effective two-particle dynamics well mimics the corresponding orbit obtained by a pair of singular points calculated by using GPE in Eq.(2). This fact justifies the validity of our trial function in Eq.(3) and the resultant equation of motion for collective coordinates in Eq.(13) and (15).

\section{B. Dynamics of three vortices}

Encouraged by the effectiveness of the collective coordinate method in the case of two vortices, we proceed to the dynamics of three vortices with the identical winding numbers $n_{1}=n_{2}=n_{3}=1$, whose Hamiltonian (15) becomes

$$
\begin{aligned}
H & =\frac{1}{2}\left(p_{x C}^{2}+p_{y C}^{2}+p_{x T}^{2}+p_{y T}^{2}+p_{x R}^{2}+p_{y R}^{2}\right) \\
& -\frac{B_{3}}{2}\left(x_{C} p_{y C}+x_{R} p_{y R}+x_{T} p_{y T}\right. \\
& \left.-y_{C} p_{x C}-y_{R} p_{x R}-y_{T} p_{x T}\right) \\
& +\left(\frac{B_{3}^{2}}{8}+\frac{B_{4}}{2}\right)\left(x_{C}^{2}+x_{R}^{2}+x_{T}^{2}+y_{C}^{2}+y_{R}^{2}+y_{T}^{2}\right) \\
& +\sum_{(i, j)=(1,2),(2,3),(3,1)} \frac{G}{\left(x_{i}-x_{j}\right)^{2}+\left(y_{i}-y_{j}\right)^{2}} \\
& \times \exp \left(-\frac{x_{i}^{2}+y_{i}^{2}+x_{j}^{2}+y_{j}^{2}}{\Delta}\right),
\end{aligned}
$$

where Jacobi coordinates $\left(x_{T}, y_{T}\right.$, etc $)$ are defined by

$$
\begin{aligned}
\left(x_{T}, y_{T}\right) & =\frac{1}{\sqrt{3}}\left(x_{1}+x_{2}+x_{3}, y_{1}+y_{2}+y_{3}\right) \\
\left(x_{C}, y_{C}\right) & =\frac{1}{\sqrt{2}}\left(x_{1}-x_{3}, y_{1}-y_{3}\right), \\
\left(x_{R}, y_{R}\right) & =\frac{1}{\sqrt{6}}\left(x_{1}+x_{3}-2 x_{2}, y_{1}+y_{3}-2 y_{2}\right) \\
\left(p_{x R}, p_{y R}\right) & =\frac{1}{\sqrt{6}}\left(p_{x 1}+p_{x 3}-2 p_{x 2}, p_{y 1}+p_{y 3}-2 p_{y 2}\right) \\
\left(p_{x T}, p_{y T}\right) & =\frac{1}{\sqrt{3}}\left(p_{x 1}+p_{x 2}+p_{x 3}, p_{y 1}+p_{y 2}+p_{y 3}\right) \\
\left(p_{x C}, p_{y C}\right) & =\frac{1}{\sqrt{2}}\left(p_{x 2}-p_{x 1}, p_{y 2}-p_{y 1}\right),
\end{aligned}
$$

which conserve the canonical structure $\left(\left\{x_{T}, p_{x T}\right\}=\right.$ $\frac{1}{3} \sum_{j=1}^{3}\left\{x_{j}, p_{x j}\right\}=1$, etc.). Here $\left(x_{T}, y_{T}\right),\left(x_{C}, y_{C}\right)$, and $\left(x_{R}, y_{R}\right)$ represent the center of mass of all three components, the relative displacement, and the bisector of the vertex $\left(x_{2}, y_{2}\right)$, respectively. In marked contrast to the massless three vortex system in 2 dimensions which is integrable, all 6 degrees of freedom are coupled and the number of independent constants of motion is 2 (energy and $z$-component of angular momentum). Then Poincaré-Bendixon's theorem guaranties the nonintegrability and chaos of the three inertial vortex system [30].

We can construct from (20) the canonical equations of motion for $x_{T}, y_{T}, x_{C}, y_{C}, x_{R}, y_{R}$ and their canonical-conjugate variables, which is solved numerically. Poincaré cross section and power spectra for the kinetic energy $E_{k}(t)$ in Fig. 2 give a clear evidence of high-dimensional chaos. Because of the short-range nature of the interaction, we see the emergence of chaos in low-lying energy regions where vortices often meet each other.
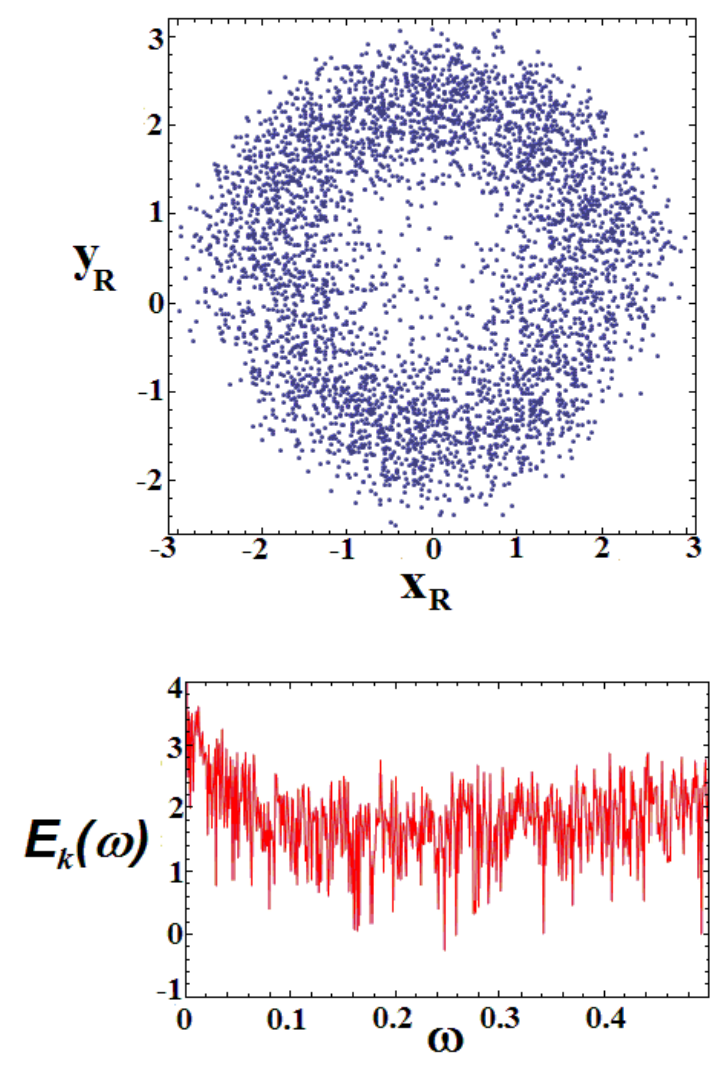

FIG. 2: Three vortices dynamics with identical winding numbers $\left(n_{1}=n_{2}=n_{3}=1\right) . \quad g_{1}=g_{2}=100$. Initial values: $x_{R}(0)=x_{C}(0)=x_{T}(0)=1, y_{R}(0)=-1, y_{C}(0)=y_{T}(0)=1$; $p_{x, R}(0)=p_{x, C}(0)=p_{x, T}(0)=p_{y, R}(0)=p_{y, C}(0)=p_{y, T}(0)=$ 1. (a) Poincaré cross section; (b) Power spectra for kinetic energy $E_{k}(t)$. 


\section{CONCLUSION}

We explored vortex dynamics in the 2-dimensional multi-component BEC in the harmonic trap in the case that each component has a single vortex. The investigation beyond the Thomas-Fermi regime is made on the nonlinear Schrödinger equation with strong repulsive cubic nonlinearity. With use of a trial vortex function based on the Padé approximation which is regularized due to a trap, we applied a collective coordinates method, obtaining an effective nonlinear dynamics for vortex cores (particles), which is equivalent to charged particles with inertia under a strong spring force and in the presence of Lorentz force with the uniform magnetic field. The inter-particle interaction is singularly-repulsive and short-ranged with its magnitude decreasing with increasing distance of the center of mass from the trapping center. The most important finding is the nonzero inertia of vortices, which is not present in the conventional theory of point vortices widely used in the usual hydrodynamics [25 29]. The system of three vortices with inertia can be chaotic, in contrast to the corresponding case of point vortices without inertia.

Acknowledgments. One of the authors (K. N.) is grateful to F. Abdullaev, B. Baizakov, S. Dmitriev and M. Tsubota for useful comments in various important stages of this work.

\section{Appendix A: Calculation of typical integrals}

We shall calculate some integrals used in this paper.

1. Integral $A \equiv \iint d x d y f_{i}^{2}\left(\dot{x}_{i} \frac{\partial \phi_{i}}{\partial x_{i}}+\dot{y}_{i} \frac{\partial \phi_{i}}{\partial y_{i}}\right)$

Substituting Eq.(3) into the above integrand, we find

$$
\begin{aligned}
A & =\frac{1}{\pi \Delta}\left(\frac{N}{N_{0}}\right)^{2} \int_{-\infty}^{\infty} \int_{-\infty}^{\infty} d x d y e^{-\frac{x^{2}+y^{2}}{\Delta}} \\
& \times\left[n_{i} \frac{\dot{x}_{i}\left(y-y_{i}\right)-\dot{y}_{i}\left(x-x_{i}\right)}{2 \xi^{2}+\left(x-x_{i}\right)^{2}+\left(y-y_{i}\right)^{2}}\right. \\
& \left.-\left(\alpha_{i} \dot{x}_{i}+\beta_{i} \dot{y}_{i}\right) \frac{\left(x-x_{i}\right)^{2}+\left(y-y_{i}\right)^{2}}{2 \xi^{2}+\left(x-x_{i}\right)^{2}+\left(y-y_{i}\right)^{2}}\right],
\end{aligned}
$$

where $N_{0}=\frac{1}{\sqrt{\pi \Delta}}$ and $N$ is the normalization constant defined below Eq.(3). Equation (A1) is a sum of $n_{i^{-}}$ dependent term $\left(A_{1}\right)$ and $\left(\alpha_{i}, \beta_{i}\right)$-dependent term $\left(A_{2}\right)$.
Below we shall concentrate on $A_{1}$ because $A_{2}$ is easily calculable. Using polar coordinates as

$$
\begin{aligned}
x-x_{i} & =\rho \cos \theta \quad x_{i}=l_{i} \cos \phi_{i} \\
y-y_{i} & =\rho \sin \theta \quad y_{i}=l_{i} \sin \phi_{i} \\
d x d y & =\rho d \rho d \theta
\end{aligned}
$$

we rewrite the integral $A_{1}$ as

$$
\begin{aligned}
A_{1} & =\frac{n_{i}}{\pi \Delta}\left(\frac{N}{N_{0}}\right)^{2} \int_{0}^{\infty} d \rho e^{-\frac{\rho^{2}+l_{i}^{2}}{\Delta}} \frac{\rho^{2}}{2 \xi^{2}+\rho^{2}} \\
& \times \int_{0}^{2 \pi} d \theta\left(\dot{x}_{i} \sin \theta-\dot{y}_{i} \cos \theta\right) e^{-\frac{2 \rho l_{i}}{\Delta} \cos \left(\theta-\phi_{i}\right)}
\end{aligned}
$$

The $\theta$-integration gives

$$
\begin{array}{r}
\int_{0}^{2 \pi} d \theta\left(\dot{x}_{i} \sin \theta-\dot{y}_{i} \cos \theta\right) e^{-\frac{2 \rho l_{i}}{\Delta} \cos \left(\theta-\phi_{i}\right)} \\
\quad=\int_{0}^{2 \pi} d \tilde{\theta}\left[\left(\dot{x} \cos \phi_{i}+\dot{y} \sin \phi_{i}\right) \sin \tilde{\theta}\right. \\
\left.+\left(\dot{x} \sin \phi_{i}-\dot{y} \cos \phi_{i}\right) \cos \tilde{\theta}\right] e^{-\frac{2 \rho l_{i}}{\Delta} \cos \tilde{\theta}} \\
=-\pi\left(\dot{x}_{i} y_{i}-\dot{y}_{i} x_{i}\right)\left(\frac{2 \rho}{\Delta}+\frac{\rho^{3} l_{i}^{2}}{\Delta^{3}}\right) .
\end{array}
$$

Here we took $\tilde{\theta}=\theta-\phi_{i}$ and used the formulas like

$$
\int_{0}^{2 \pi} d \tilde{\theta} \cos \tilde{\theta} e^{-z \cos \tilde{\theta}}=-2 \pi I_{1}(z),
$$

where $I_{1}(z)$ is the modified Bessel function, which is expanded as

$$
I_{1}(z)=\frac{z}{2}+\frac{z^{3}}{16}+O\left(z^{5}\right) .
$$

As for $\rho$-integration, we use identities like

$$
\frac{\rho^{3}}{2 \xi^{2}+\rho^{2}}=\rho-\frac{2 \xi^{2} \rho}{2 \xi^{2}+\rho^{2}}
$$

and then apply the formulas

$$
\int_{0}^{\infty} d \rho \rho^{n} e^{-\frac{\rho^{2}}{\Delta}}=\frac{1}{2} \Delta^{\frac{n+1}{2}} \Gamma\left(\frac{n+1}{2}\right)
$$

and

$$
\begin{aligned}
& \int_{0}^{\infty} d \rho e^{-\frac{\rho^{2}}{\Delta}} \frac{\rho}{2 \xi^{2}+\rho^{2}}=\frac{1}{2} e^{\frac{2 \xi^{2}}{\Delta}} \Gamma\left(0, \frac{2 \xi^{2}}{\Delta}\right) \\
= & \frac{1}{2} e^{\frac{2 \xi^{2}}{\Delta}}\left(-\gamma-\ln \frac{2 \xi^{2}}{\Delta}+\frac{2 \xi^{2}}{\Delta}-\left(\frac{\xi^{2}}{\Delta}\right)^{2}\right) .
\end{aligned}
$$


$\Gamma(0, z) \equiv \int_{z}^{\infty} t^{-1} e^{-t} d t$ above is the incomplete gamma function of the second kind and can be expanded as

$$
\Gamma(0, z)=-\gamma-\ln z+z-\frac{z^{2}}{4}+\frac{z^{3}}{18}+O\left(z^{4}\right) .
$$

The final result is

$$
A_{1}=-\left(\frac{N}{N_{0}}\right)^{2} \frac{n_{i}}{\Delta}\left(\dot{x}_{i} y_{i}-\dot{y}_{i} x_{i}\right) e^{-\frac{l_{i}^{2}}{\Delta}}\left(d_{1}+\frac{d_{2} l_{i}^{2}}{\Delta}\right),
$$

where $d_{1}$ and $d_{2}$ are listed in Table 1. Equation (A11), combined with

$$
A_{2}=-\left(\frac{N}{N_{0}}\right)^{2}\left(\alpha_{i} \dot{x_{i}}+\beta_{i} \dot{y_{i}}\right) d_{1},
$$

gives rise to the calculated result for $A$ in Eq. (A1).

$$
\begin{gathered}
\text { 2. Integral } B \equiv \frac{g_{1}}{2} \iint f_{i}^{4} d x d y \\
B=\frac{g_{1}}{2 \pi^{2} \Delta^{2}}\left(\frac{N}{N_{0}}\right)^{4} \int_{-\infty}^{\infty} \int_{-\infty}^{\infty} d x d y e^{-\frac{2\left(x^{2}+y^{2}\right)}{\Delta}} \\
\times\left(\frac{\left(x-x_{i}\right)^{2}+\left(y-y_{i}\right)^{2}}{2 \xi^{2}+\left(x-x_{i}\right)^{2}+\left(y-y_{i}\right)^{2}}\right)^{2}
\end{gathered}
$$

Using polar coordinates in (A2), we rewrite the integral as

$$
\begin{aligned}
B & =\frac{g_{1}}{2 \pi^{2} \Delta^{2}}\left(\frac{N}{N_{0}}\right)^{4} \int_{0}^{\infty} \rho d \rho e^{-\frac{2\left(\rho^{2}+l_{i}^{2}\right)}{\Delta}} \\
& \times\left(\frac{\rho^{2}}{2 \xi^{2}+\rho^{2}}\right)^{2} \int_{0}^{2 \pi} d \theta e^{-\frac{4 \rho l_{i}}{\Delta} \cos \left(\theta-\phi_{i}\right)}
\end{aligned}
$$

First, we carry out the $\theta$-integration. Note that

$$
\int_{0}^{2 \pi} d \theta e^{-z \cos \theta}=2 \pi I_{0}(z)
$$

where $I_{0}(z)$ is the modified Bessel function, which is expanded as

$$
I_{0}(z)=1+\frac{z^{2}}{4}+\frac{z^{4}}{64}+O\left(z^{6}\right) .
$$

Concerning the $\rho$-integration, we first employ the decomposition

$$
\frac{\rho^{4}}{\left(2 \xi^{2}+\rho^{2}\right)^{2}}=1-\frac{4 \xi^{2}}{2 \xi^{2}+\rho^{2}}+\frac{4 \xi^{4}}{\left(2 \xi^{2}+\rho^{2}\right)^{2}},
$$

and then apply the formulas like

$$
\int_{0}^{\infty} d \rho \rho e^{-\frac{2 \rho^{2}}{\Delta}} I_{0}\left(\frac{4 \rho l_{i}}{\Delta}\right)=\frac{\Delta}{4} e^{\frac{2 l_{i}^{2}}{\Delta}} .
$$

The final result is

$$
B=\left(\frac{N}{N_{0}}\right)^{4} \frac{g_{1}}{\pi \Delta}\left[\frac{1}{4}+d_{7} e^{-\frac{2 l_{i}^{2}}{\Delta}}\right],
$$

where $d_{7}$ is given in Table 1 .

\section{Appendix B: Calculation of inter-vortices interaction $U$}

This interaction is due to the integral

$$
\begin{aligned}
U & \equiv g_{2} \int_{-\infty}^{\infty} \int_{-\infty}^{\infty} f_{i}^{2} f_{j}^{2} d x d y \\
& =\frac{g_{2}}{\pi^{2} \Delta^{2}}\left(\frac{N}{N_{0}}\right)^{4} \int_{-\infty}^{\infty} \int_{-\infty}^{\infty} d x d y e^{-\frac{2\left(x^{2}+y^{2}\right)}{\Delta}} \\
& \times \frac{\left(x-x_{i}\right)^{2}+\left(y-y_{i}\right)^{2}}{2 \xi^{2}+\left(x-x_{i}\right)^{2}+\left(y-y_{i}\right)^{2}} \\
& \times \frac{\left(x-x_{j}\right)^{2}+\left(y-y_{j}\right)^{2}}{2 \xi^{2}+\left(x-x_{j}\right)^{2}+\left(y-y_{j}\right)^{2}} .
\end{aligned}
$$

With use of a prescription $U \equiv \frac{g_{2}}{\pi^{2} \Delta^{2}}\left(\frac{N}{N_{0}}\right)^{4} \hat{U}$, the integral becomes:

$$
\begin{aligned}
& \hat{U}=\int_{-\infty}^{\infty} \int_{-\infty}^{\infty} d x d y e^{-\frac{2\left(x^{2}+y^{2}\right)}{\Delta}} \\
& \times\left[1-2 \xi^{2}\left(\frac{1}{2 \xi^{2}+\left(x-x_{i}\right)^{2}+\left(y-y_{i}\right)^{2}}+(i \rightarrow j)\right)\right. \\
& \left.+\frac{4 \xi^{4}}{\left(2 \xi^{2}+\left(x-x_{i}\right)^{2}+\left(y-y_{i}\right)^{2}\right)(i \rightarrow j)}\right] \\
& \equiv \hat{U}_{0}+\hat{U}_{2}+\hat{U}_{4} .
\end{aligned}
$$

$\hat{U}_{0}, \hat{U}_{2}$ and $\hat{U}_{4}$ corresponds to the contributions from $O\left(\xi^{0}\right), O\left(\xi^{2}\right)$ and $O\left(\xi^{4}\right)$, respectively. Among them, $\hat{U}_{4}$ is responsible to the vortex-vortex interaction, which we shall calculate below.

Let's define the center-of-mass and relative coordinates by

$$
x_{G}^{i j}=\frac{x_{j}+x_{i}}{2} ; \quad y_{G}^{i j}=\frac{y_{j}+y_{i}}{2}
$$




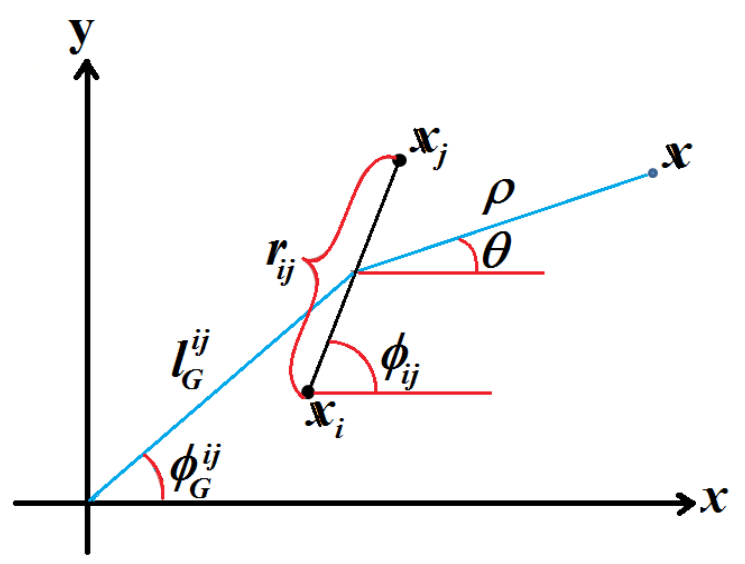

FIG. 3: New integration variables, center-of-mass and relative coordinates.

and

$$
x_{i}-x_{j}=r_{i j} \cos \phi_{i j} ; \quad y_{i}-y_{j}=r_{i j} \sin \phi_{i j},
$$

respectively, and transform the integration variables to new ones as (see Fig 3)

$$
\begin{aligned}
x-x_{G}^{i j} & =\rho \cos \theta, \quad x_{G}^{i j}=l_{G}^{i j} \cos \phi_{G}^{i j} \\
y-y_{G}^{i j} & =\rho \sin \theta, \quad y_{G}^{i j}=l_{G}^{i j} \sin \phi_{G}^{i j} \\
d x d y & =\rho d \rho d \theta .
\end{aligned}
$$

Then $\hat{U}_{4}$ in Eq. (B2) becomes

$$
\begin{aligned}
\hat{U}_{4} & =\int_{0}^{\infty} \rho d \rho \int_{0}^{2 \pi} d \theta^{\prime} e^{\frac{-2}{\Delta}\left(\left(l_{G}\right)^{2}+\rho^{2}+2 \rho l_{G}^{i j} \cos \left(\theta^{\prime}+\phi_{i j}-\phi_{G}^{i j}\right)\right)} \\
& \times\left[\frac{4 \xi^{4}}{\left(2 \xi^{2}+\rho^{2}+\frac{r_{i j}^{2}}{4}\right)^{2}-\rho^{2} r_{i j}^{2} \cos ^{2}\left(\theta^{\prime}\right)}\right],
\end{aligned}
$$

where we moved to a new angle variable $\theta^{\prime} \equiv \theta-\phi_{i j}$. Using the expansion

$$
e^{-X \cos \left(\theta^{\prime}+\alpha\right)}=\sum_{n=-\infty}^{\infty}(-1)^{n} I_{n}(X) e^{i n \alpha} e^{i n \theta^{\prime}}
$$

in Eq. (B6) and keeping the $n=0$ term, the integration over the angle variable leads to:

$$
\begin{aligned}
& \int_{0}^{2 \pi} d \theta^{\prime} \frac{4 \xi^{4}}{\left(2 \xi^{2}+\rho^{2}+\frac{r_{i j}^{2}}{4}\right)^{2}-\rho^{2} r_{i j}^{2} \frac{1+\cos 2 \theta^{\prime}}{2}} \\
= & \frac{8 \pi \xi^{4}}{2 \xi^{2}+\rho^{2}+\frac{r_{i j}^{2}}{4}} \\
\times & \frac{1}{\sqrt{\left(2 \xi^{2}+\left(\rho-\frac{r_{i j}}{2}\right)^{2}\right)\left(2 \xi^{2}+\left(\rho+\frac{r_{i j}}{2}\right)^{2}\right)}},
\end{aligned}
$$

where we used the formula $\int_{0}^{2 \pi} d \theta \frac{1}{c+b \cos \theta}=\frac{2 \pi}{\sqrt{(c-b)(c+b)}}$.

We shall proceed to $\rho$ integration. Here we should note: In the case $\xi \ll 1$, the Lorentzian-like function on r.h.s. of Eq. (B8) is sharply peaked around $\rho=\frac{r_{i j}}{2}$ and is well approximated for $\rho>0$ by Gaussian, $\frac{8 \sqrt{2} \pi \xi^{3}}{r_{i j}^{3}} \exp \left(-\frac{\left(\rho-\frac{r_{i j}}{2}\right)^{2}}{4 \xi^{2}}\right)$. Therefore, the $\rho$ integration leads to:

$$
\begin{aligned}
\hat{U}_{4} & =\frac{8 \sqrt{2} \pi \xi^{3}}{r_{i j}^{3}} e^{-\frac{2\left(l_{G}\right)^{2}}{\Delta}} \\
& \times\left[\int_{0}^{\infty} \rho e^{-\frac{2 \rho^{2}}{\Delta}} I_{0}\left(\frac{4 \rho l_{G}^{i j}}{\Delta}\right) \exp \left(-\frac{\left(\rho-\frac{r_{i j}}{2}\right)^{2}}{4 \xi^{2}}\right) d \rho\right] \\
& =\frac{8 \pi^{3 / 2} \xi^{4}}{\left(1+\xi^{2} / \Delta\right)\left(1+8 \xi^{2} / \Delta\right)^{1 / 2}} \frac{1}{r_{i j}^{2}} I_{0}\left(\frac{2 l_{G}^{i j} r_{i j}}{\Delta\left(1+2 \xi^{2} / \Delta\right)}\right) \\
& \times \exp \left(-\frac{2 l_{G}^{2}}{\Delta}\right) \exp \left(-\frac{r_{i j}^{2}}{2 \Delta\left(1+8 \xi^{2} / \Delta\right)}\right),
\end{aligned}
$$

where the integration was carried out by the saddle-point approximation which is justified in the case $\xi \ll 1$. Since each vortex dynamics occurs in the range $\frac{2 l_{G}^{i j} r_{i j}}{\Delta} \ll 1$ because of the notion above Eq.(16) in Sec II , we may approximate $I_{0}\left(\frac{2 l_{G}^{i j} r_{i j}}{\Delta\left(1+2 \xi^{2} / \Delta\right)}\right) \sim 1$ and neglect the contributions from $I_{n}(x)$ with $n=1,2 . \cdots$ in the region $x \ll 1$. Then we reach

$$
\begin{aligned}
U_{4} & \equiv U_{4}\left(r_{i j}, l_{G}^{i j}\right) \equiv \frac{g_{2}}{\pi^{2} \Delta^{2}}\left(\frac{N}{N_{0}}\right)^{4} \hat{U}_{4} \\
& \approx \frac{8 g_{2} \xi^{4}}{\pi^{1 / 2} \Delta^{2}} \frac{1}{r_{i j}^{2}} \exp \left(-\frac{r_{i j}^{2}+4\left(l_{G}^{i j}\right)^{2}}{2 \Delta}\right)
\end{aligned}
$$

with use of $\left(\frac{N}{N_{0}}\right)^{4} \approx 1$. At first the magnitude of the inter-vortices interaction looks very small (i.e., $O\left(\xi^{4}\right)$ ), but, after scaling to make unity the inertial mass of each vortex, it becomes $O\left(g_{2}\right)$ (see Eq.(17)). It is interesting that the inter-vortices interaction energy in a multicomponent BEC with no trap estimated in a different approximation (i.e., under the Abrikosov ansatz) is also proportional to $\frac{1}{r_{i j}^{2}}$ in the asymptotic region [31].

Finally we note the remaining contributions, $\hat{U}_{0}+\hat{U}_{2}$, in Eq.(B2). Their integration is quite simple and gives rise to

$$
\begin{aligned}
U_{0} & +U_{2} \equiv \frac{g_{2}}{\pi^{2} \Delta^{2}}\left(\frac{N}{N_{0}}\right)^{4}\left(\hat{U}_{0}+\hat{U}_{2}\right) \\
& \approx \frac{g_{2}}{\pi \Delta}\left(\frac{N}{N_{0}}\right)^{4}\left[\frac{1}{2}+2 \frac{\xi^{2}}{\Delta}\left(\gamma+\ln \frac{2 \xi^{2}}{\Delta}\right)\left(e^{-\frac{l_{i}^{2}}{\Delta}}+e^{-\frac{l_{j}^{2}}{\Delta}}\right)\right] .
\end{aligned}
$$


In the final expression, the first term is constant, giving no contribution to the dynamics in Eq.(13), and the second one is a sum of single-particle contributions of
$O\left(\frac{\xi^{2}}{\Delta}\right)$ which renormalizes the 7 -th line in Eq.(8), giving no substantial contribution to Eq.(13).
[1] C.J. Pethick and H. Smith, Bose-Einstein Condensation in Dilute Gases (Cambridge Univ. Press, Cambridge, 2002).

[2] L.P. Pitaevskii and S. Stringari Bose-Einstein Condensation (Oxford Univ. Press, Oxford, 2003).

[3] P.G. Kevrekidis, D.J. Frantzeskakis and R. CarreteroGonzález Emergent Nonlinear Phenomena in BoseEinstein Condensates (Springer-Verlag, Berlin, 2008).

[4] A.D. Martin, C.S. Adams and S.A. Gardiner, Phys. Rev. Lett. 98, 020402 (2007).

[5] V.M. Pérez-García, Physica D 191, 211 (2004); G.D. Montesinos,V.M. Pérez-García and H. Michinel, Phys. Rev. Lett. 92, 133901-1(2004); V. M. Pérez-García, et al., Physica D238, 1289 (2009).

[6] H. Yamasaki, Y. Natsume and K. Nakamura, J. Phys. Soc. Jpn. 74, 1887 (2005).

[7] T.W. Neely et al., Phys. Rev. Lett. 104, 160401 (2010).

[8] D. V. Freilich, et al. Science 329, 1182 (2010).

[9] S. Middlkamp, et al., Phys. Rev. A84, 011605(R) (2011).

[10] G. Thalhammer, et al., Phys. Rev. Lett. 100, 210402 (2008).

[11] S. B. Papp, et al., Phys. Rev. Lett. 93, 040402 (2008).

[12] S. Tojo, et al., Phys. Rev. A 82, 033609 (2010).

[13] A.L. Fetter and A.A. Svidzinsky, J. Phys.: Condens. Matter 13 R135 (2001).

[14] A.L. Fetter, Rev. Mod. Phys. 81647 (2009) .

[15] P.J. Torr, et al., Phys. Lett. A375, 3004 (2011).

[16] J.C. Neu, Physica D 43, 385 (1990).

[17] A. Aftalion, Vortices in Bose-Einstein Condensates
(Birkhäuser, Boston, 2006).

[18] K. Sasaki, N. Suzuki and H. Saito, Phys. Rev. Lett. 104, 150404 (2010).

[19] K. Nakamura, Prog. Theor. Phys. Suppl. 166, 179 (2007).

[20] I. Aranson and V. Steinberg, Phys. Rev. B 53, 75 (1996).

[21] N. G. Berloff, J. Phys. A 37, 1617 (2004).

[22] J.J. García-Ripoll et al., Phys. Rev. Lett. 84, 4264 (2000).

[23] J.J. García-Ripoll et al., Phys. Rev. Lett. 87, 140403 (2001).

[24] L.C. Crasovan et al., Phys. Rev. A 68, 063609 (2003).

[25] H. Lamb, Hydrodynamics (Cambridge Univ. Press, Cambridge, 1967).

[26] L. Onsager, Nuovo Cimento Supple. 6, 279 (1949).

[27] G.K. Batcheor, An Introduction to Fluid Dynamics (Cambridge Univ. Press, Cambridge, 1967).

[28] U. Frisch, Turbulence: The Legacy of A. N. Kolmogorov (Cambridge Univ. Press, Cambridge, 1995).

[29] J. Roenby and H. Aref, Proc. R. Soc. A 466, 1871 (2010).

[30] In the usual theory of hydrodynamics, interacting point vortices in 2 dimensions have 3 independent constants of motion, i.e., total energy, $x$-(or $y$-) component of momentum and $z$-component of angular momentum. Therefore the number of vortices must be larger than 3 , to make the system non-integrable and chaotic.

[31] M. Eto, K. Kasamatsu, M. Nitta, H. Takeuchi and M. Tsubota, Phys. Rev. A 83, 063603 (2011). 